\title{
Assessment of the Students' Physical Fitness Level and Metrological Justification of Motive Tests
}

\author{
Nadezhda I. Palagina ${ }^{1}$, Michael M. Polevshchikov ${ }^{1}$, Yulia A. Dorogova ${ }^{1}$, Maria L. Blinova ${ }^{1}$, Andrey V. \\ Zakamsky $^{1}$, Aleksandr M. Shraga ${ }^{1} \&$ Eleonora A. Loskutova ${ }^{1}$ \\ ${ }^{1}$ Mari State University, Yoshkar-Ola, Russia \\ Correspondence: Yulia A. Dorogova, Faculty of Physical Culture, Sports and Tourism, Mari State University, 1 \\ Lenin Square, 424000, Yoshkar-Ola, Russia. Tel: 7-836-245-4389. E-mail: julya_dorogova@rambler.ru
}

\author{
Received: February 21, 2015 Accepted: March 15, 2015 Online Published: April 29, 2015 \\ doi:10.5539/res.v7n8p137 URL: http://dx.doi.org/10.5539/res.v7n8p137
}

\begin{abstract}
In this article there is considered the problem of an assessment of the students' physical fitness level, which aren't playing sports. Using of such assessment in the course of students' physical training will allow to consider specific features of students, to define their weak and strengths and by that to provide their interest in improvement of their physical state's level. The assessment of physical fitness level of the studying youth also promotes to the involvement of student's youth in the self-improvement process and it helps to increase their physical fitness level. In order that such assessment was objective and real, it is necessary to carry out metrological justification of the test tasks entering to a complex. Metrological justification includes determination of coefficient of reliability and informational content of the offered test tasks in order that they could be used for an assessment of the separate parties of physical fitness. The factorial analysis of the offered tests is necessary to define possibilities of these tests to estimate this or that physical quality. In this article there are represented the results of the metrological assessment of test "battery", that consists of the next 5 motive tasks: 10 minutes running; lifting of a trunk in 30 seconds lying on a back, hands along a trunk, feet aren't fixed; an inclination forward, standing on a bench; bending of hands (push-up from a floor) in an emphasis on a lap in 30 seconds; transferring of a gymnastic stick straight arms for a back.
\end{abstract}

Keywords: physical fitness, motive tests, method of the factorial analysis, reliability coefficient, informational content coefficient

\section{Introduction}

At the present stage of development and improvement of the physical training system of the higher school, the special relevance is gained by an assessment of physical fitness level of the studying youth. It allows considering specific features of students, their abilities and interests at occupations by physical culture. Physical trainings are urged to solve all problems of the students' physical education. They help to interest young people in increasing of their physical fitness level, to influence on the functionality of an organism, taking into account their specific features.

So far, in the activities of university for the students' physical training, there is absented the accounting of specific features of each student and in this regard a lack of systematic growth of physical fitness' indicators(Akchurin, 1996). An important problem there is also a problem of involvement of student's youth in process of physical self-improvement for the purpose of the physical fitness' improvement (Palagina \& Blinova, 2010). For finding the solution of this problem it is possible to do a research in the new sports and improving technologies, providing an application of a wide arsenal of physical culture means, taking into an account specific features of each person. However in the practice course of physical training in the university it became clear that most of students aren't engaged in physical exercises independently that is explained by existence of the following reasons (Vinogradov, 1996):

1) The absence at the student's age of biogenous needs for the movement, which, for example, is observed at the children's age.

2) The absence of social needs for the physical form improvement because of the weak statement of the physical education in preschool and school institutions. 
3) The deficiency of time, the lack of conditions or bigger interest to the inactive types of activity in free time.

Therefore, the increasing of the students' physical fitness is still depended on the class periods. In this case the paramount values have the content of occupations and the quality of their carrying out. As one of possible options of successful realization of the students' active inclusion in the process of sports self-education, can be taken such form of the educational process organization, according to which the practical training and the training program would be supplemented with vigorous creative work of self-education and physical self-improvement. For this purpose, under the leadership of the teacher, each student could develop the individual program of physical self-improvement including recommendations about selection and a performance technique of complexes of physical exercises, about a dosage of loading and about criteria of the assessment defining efficiency of their performance, taking into account a functional and somatic state those, who are engaged.

The assessment of the physical fitness level and motive abilities of the student's youth allows to realize own motive skills (Hasin \& Rafalovich, 1995; Hasin, Gromyko, \& Rafalovich, 1999; Palagina, 2013). It gives a chance to define the level of the motive qualities development, and also the purposes and ways of the physical self-improvement. All this promotes active involvement of students in this process. Thus, the assessment of physical fitness level helps to interest young people in increasing of the physical fitness level, effectively to influence functionality of an organism taking into account their specific features.

The assessment of motive (motor) abilities of the person allows exercising control over the physical fitness level and over the system effectiveness of physical preparation. Motor tests represent the motive tasks allowing to define a condition of motive functions of the person: a) the level of physical qualities; b) the opportunity to carry out a certain circle of movements (motive abilities, skills).

For an assessment of the physical fitness level of the studying youth there are used some motive tests or their complexes at which performance it is possible to receive information on motive abilities of the engaged. The results of the motive tests implementation allow to reveal weak and strengths of physical fitness of the person, and also to introduce some amendments in the management and planning of educational and training process taking into account the features of those, who are engaged.

The offered technique of planning provides identification of weak and strengths of physical fitness, i.e. the determination of specific features in development of motive abilities, and also a choice of the selective impact means on the lagging behind parties. It provides the differentiated and individualized planning of loading on the academic class in physical culture.

The need of application of the differentiated approach on educational and training occupations with students in higher education institution is caused by heterogeneity of physical fitness between the students' majority. Especially it concerns the female. There are almost $50 \%$ of girls in the university and they have the most various initial level of development of the majority of indicators. So the difference between the extreme values in indicators of the motive qualities development, within one medical group, reaches $22 \%$. This feature is characteristic, first of all, for female students of the main educational office that causes, naturally, various dynamics of their development in the course of physical preparation (Kul'kov, 1980; Yushkov, 1991; Akchurin, 1996).

Besides, the work experience on physical training of girls shows that with the help of traditional methods it is impossible to solve successfully the tasks that are set for physical culture. The standard technique of physical education in the university leads to the positive, but to the statistically doubtful gain of the results in the majority of physical development and physical fitness indicators. Such changes can't be considered satisfactory, especially for girls.

In the girls' physical education must be solved, besides the general, the specific problems based, first of all, on the knowledge of a female organism features. At the organization of occupations by physical culture with girls should to be taken into account the anatomic and physiological features of their organism. These features cause the forms of the main motive qualities manifestation - the muscular force, speed of movements, dexterity and endurance, and also define physical working capacity (Gadzhiyev, 1986). Considering the biological features of an organism, the specialists in physical education have to show care at a choice of means and methods for occupations with the female contingent.

The features of a female organism define the opportunities in some separate groups of physical exercises (Lapshina, 1989). There are no restrictions for development of dexterity and high coordination of movements by women. Smoothness of movements and big flexibility of a backbone allow the woman to surpass the man in 
plasticity, rhythmic and dancing art. At the heart of the observed plasticity of movements there is a very valuable ability to thin regulation of various degrees of muscular tension and ability randomly to regulate them. Therefore in the physical preparation there should be allocated enough places for sports occupations with inclusion of smooth and dancing movements. There is also noted the beneficial influence of rhythmic gymnastics and other types of the acyclic exercises which are carried out under music. At a choice of physical exercises for women it is necessary to consider features of motive activity peculiar to them. Therefore it is expedient to use more widely exercises of dynamic character with a big amplitude of movements, frequent change of poses and rhythmic alternation of phases of reduction and relaxation of muscles.

The performance of such exercises is connected with alternation of nervous processes in bark of big hemispheres and is followed by involvement in work of a large number of muscular groups. According to this fact there is reached the improvement of speed and coordination of actions in many links of the motive device and plasticity, grace and necessary force of movements. At the same time also should be used the power exercises in sports classes with girls, as an optimum development of muscular force and endurance is necessary for harmonious development, overcoming of vital difficulties, successful activity on a workplace and in life (the link to the article).

Thus, in connection with existence of distinctions in a structure and functions of a man's and female organism, the technique of application of physical exercises and sports activities during the work with women has to have specific character. Therefore in this research the skilled and experimental work was carried out only with girls, as a condition of the differentiated approach in physical training of students. The individualization of educational and training process is provided in the form of the differentiated approach to the engaged in the course of group occupations and in the form of individual implementation of physical training process on the basis of results on estimation of physical fitness level (Kul'kov, 1980; Kuzovenkov \& Yermylov, 1982; Kustov, 1988).

By drawing up the test complexes by definition and an assessment of the students physical fitness in the different countries this issue was resolved ambiguously. The problem of testing and estimation of physical fitness of a person and in particular student's youth didn't receive the unambiguous decision so far. Nevertheless, the researchers saved up the huge material on use of various motive tests for an assessment of the separate parties of physical fitness. According to the analysis of the applied tests or test combinations, it is possible to draw a conclusion that there are no such tests which would characterize a level of development of separate motive qualities in pure form. Therefore the assessment of the data, which was obtained as a result of application of this or that test that was chosen for determination of concrete motive quality can be accepted with a certain convention. There is no accurate clarity on the question what of the applied tests will give the greatest information on the development level of these or those parties of physical fitness of the studying youth. On the basis of these conclusions there was recommended a selection of tests for an assessment of motive abilities to carry out on a scientific basis. As measures for selection of test tasks in test "battery" it was offered to use (Vinogradov, 1996; Smirnov, Polevshchikov, 2000; Palagina \& Polevshchikov, 2014):

1) A method of the factorial analysis to estimate possibilities of the used tests, to estimate the level of the main motive qualities;

2) Determination of the reliability coefficients and informational content of motive exercises and compliance to their standards for their use as tests by definition of the development level of a certain physical quality.

\section{Methods}

The selection of test tasks in a test combination was carried out on the basis of the analysis of the existing tests for an assessment of the students' physical fitness level.

The metrological justification of the chosen motive tests for an assessment of the students' motive abilities was carried out with using by parametrical methods of mathematical statistics on a big selection with application of standard programs of the correlation analysis. The program of calculations included: the definition of an arithmetic average, the method of the factorial analysis, the determination of reliability coefficients and informational content (Bondarevskiy, 1983; Smirnov \& Polevshchikov, 2000; Palagina \& Polevshchikov, 2014).

The arithmetic average was determined according to a formula for not grouped data, because the calculation accuracy of the raw data is always higher:

$$
(\mathbf{X})=\sum X \mathbf{i} / \mathbf{N}
$$

where $(\mathrm{X})$ - an arithmetic average, $\sum$ - a summation sign, $\mathrm{X}$ - - options, $\mathrm{N}$-number of supervision.

For determining the coefficient of the offered tests' reliability there was used the retest method and determined 
the interclass coefficient of correlation ( $\left.\mathbf{r}^{\mathbf{y}} \mathbf{t} \mathbf{t}\right)$.

By the determination of the tests informational content there was calculated the coefficient of multiple correlation (r) and the coefficient of correlation by the compound criterion (rt).

The knowledge of the reliability coefficient size is necessary for the following reasons:

1) A small reliability reduces efficiency of estimates of average sizes according to selective data (accuracy of an assessment is proportional to reliability coefficient);

2) An insufficient reliability of the test doesn't allow to receive high sizes of correlation of dependences because the highest indicator happens in case of the test's correlation with itself. By the undependable test the upper bound of the correlation's coefficient is defined not with \pm 1.00 anymore, but with an indicator of its reliability;

3) The value of the reliability coefficient allows to calculate the so-called true size of correlation, having entered the amendment on reliability. As a result of such amendment the insignificant coefficient of correlation can be turned in a very big one, if the test's reliability is small.

In the course of research for determination of the reliability coefficient of the offered tests there was applied the retesting method (Bondarevskiy, 1983; Palagina, 2006).

The best measure of the test's reliability is the interclass coefficient of correlation and therefore the reliability of the offered tests of physical fitness for an assessment of motive abilities was decided on the help of so-called interclass coefficient of correlation (r'tt). In this research the coefficient of the interclass correlation was calculated between the 1 st and the 3rd attempts. The previously carried out tests showed the high degree of the offered tests' reliability.

The practical expediency of the tests' verification, which is used for an assessment of motive abilities of the studying youth, admits by all the experts for informational contents, as it allows estimating efficiency of the used tests. At the determination of the tests' informational content, there is, as a rule, a difficulty in a criterion choice. There are various approaches in the solution of this problem.

Due to the lack of the single measured criterion for determination of informational content of the offered tests there were used the following methodical receptions.

First of all there were chosen some situations, when as a predictive variables there is performed not one, but the group of the tests and for determination of informational content there was calculated the coefficient of multiple correlation (r) in which size there was estimated an efficiency of the applied tests.

The following reception was consisted in an attempt to create the compound criterion. For this purpose we were used the S-scale for transferring each result in any of tests to-point system. In this case the compound criterion (rt) is represented in the form of the sum of results on the each test (Palagina, 2006).

The factorial analysis (Godik \& Zatsiorsky, 1965; Palagina, 2006) was carried out on the materials of the correlation analysis of data of preliminary experiment on the five offered tests, including data of anthropometrical measurements (weight, growth).

The anthropometrical measurements were held for the purpose of detection of the testing results' dependence from weight and growth of the engaged.

It is considered that the individual approach in an assessment of a condition of the people's physical fitness can't be objective without their morphological features. The existing experimental data testify to existence of dependences between growth-weight indicators of examinees and various forms of their motive abilities. It is established that the body weight influences on the result in pulling up, and at the same time neither weight, nor length of a body have no essential impact on time of passing of a distance. It is known that from two anthropometrical signs (weight and growth), the body weight has the greatest impact on sports result. Some experts consider that the indicators that are characterizing the total sizes of a body (growth and especially weight), statistically authentically influence on the sizes of the maximum and partially high-speed force.

For the metrological justification of the tests' offered complex for an assessment of the female students' motive abilities, there was used the mass testing of female students in which took part 954 girls of the higher education institutions of Yoshkar-Ola city (Palagina, 2005; Palagina, 2006).

\section{Results}

There were entered the created test complex five motive tasks: 1) 10 min. running; 2) lifting of a trunk for $30 \mathrm{sec}$. lying on a back, hands along a trunk, feet aren't fixed; 3) an inclination forward, standing on a bench; 4) bending of hands (push-up from a floor) in an emphasis on a lap for $30 \mathrm{sec}$; 5) transferring of a gymnastic stick straight 
arms for a back (Palagina, 2005; Palagina, 2006).

The results of the metrological calculations for the recommended test tasks are presented in the tables 1,2 and 3 (Palagina, 2005).

Table 1. The results of the tests' metrological justification

\begin{tabular}{|c|c|c|c|c|c|}
\hline No & test & $\begin{array}{l}\text { arithmeticaver } \\
\text { age } \\
(\mathrm{X})\end{array}$ & $\begin{array}{l}\text { reliabilitycoef } \\
\text { ficient }\left(r^{\prime} t t\right)\end{array}$ & $\begin{array}{l}\text { multiplecoefficie } \\
\text { ntofcorrelation } \\
\text { ( })\end{array}$ & $\begin{array}{l}\text { correlation } \\
\text { coefficient by } \\
\text { compound } \\
\text { criterion (rt) }\end{array}$ \\
\hline 1 & 10 min. running (meters) & 1700 & 0.900 & 0.905 & 0.850 \\
\hline 2 & $\begin{array}{l}\text { lifting of a trunk for } 30 \mathrm{sec} \text {. } \\
\text { (modified) } \\
\text { (number of repetitions) }\end{array}$ & 18 & 0.904 & 0.880 & 0.827 \\
\hline 3 & $\begin{array}{l}\text { inclination forward, standing } \\
\text { on a gymnastic bench }(\mathrm{cm})\end{array}$ & +14 & 0.975 & 0.377 & 0.515 \\
\hline 4 & $\begin{array}{l}\text { Push-up in } 30 \text { seconds from } \\
\text { the situation "emphasis lying } \\
\text { on a lap" } \\
\text { (numberofrepetitions) }\end{array}$ & 21 & 0.951 & 0.815 & 0.805 \\
\hline 5 & $\begin{array}{l}\text { transferring of a gymnastic } \\
\text { stick straight arms for a back }\end{array}$ & 30 & 0.978 & 0.353 & 0.347 \\
\hline
\end{tabular}

Table 2. Correlation dependences between indicators of the female students' testing who aren't doing sports ( $\mathrm{n}=$ 954)

\begin{tabular}{|c|c|c|c|c|c|c|c|c|c|}
\hline No & Indicators & 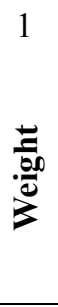 & 苋 & 을 & 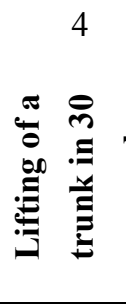 & 串 & 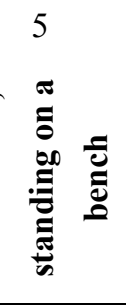 & 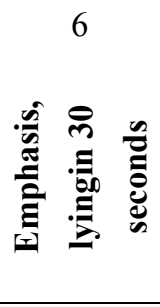 & 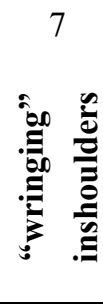 \\
\hline 1 & Weight & 1 & 0.519 & -0.340 & -0.261 & & -0.081 & -0.275 & -0.020 \\
\hline 2 & Growth & & 1 & -0.057 & -0.008 & & 0.001 & -0.085 & 0.004 \\
\hline 3 & 10 minutesrunning & & & 1 & 0.869 & & 0.150 & 0.834 & 0.023 \\
\hline 4 & $\begin{array}{l}\text { Lifting of a trunk in } \\
30 \text { seconds }\end{array}$ & & & & 1 & & 0.137 & 0.797 & 0.018 \\
\hline 5 & $\begin{array}{l}\text { Inclination, standing } \\
\text { on a bench }\end{array}$ & & & & & & 1 & 0.148 & -0.331 \\
\hline 6 & $\begin{array}{l}\text { Emphasis, lyingin } 30 \\
\text { seconds }\end{array}$ & & & & & & & 1 & 0.059 \\
\hline 7 & $\begin{array}{l}\text { "wringing" } \\
\text { shoulders }\end{array}$ & & & & & & & & 1 \\
\hline
\end{tabular}


Table 3. Factorial weight, allocated as a result of the correlation analysis

\begin{tabular}{ccc}
\hline Number of a factor & Percentage of factors in selection & Total dispersion of selection \\
\hline I & 41.06 & 41.06 \\
II & 19.95 & 61.01 \\
III & 18.81 & 79.82 \\
IV & 9.27 & 89.09 \\
V & 6.33 & 95.42 \\
VI & 2.85 & 98.27 \\
VII & 1.73 & 100.00 \\
\hline
\end{tabular}

\section{Discussion}

The analysis of test tasks allowed to assume that this test "battery" rather fully characterizes the physical fitness level of the student's youth. The offered tests cover a wide range of physiological systems, mechanisms and motive abilities of a person. Besides, the performance of the offered exercises demands the high level of functioning of various physiological systems and mechanisms of energy, and also work of various muscles' groups at the various modes of muscular contractions and motive abilities of a person.

So the 10 minutes running imposes certain requirements to aerobic opportunities of an organism, to a condition of cardio respiratory and hormonal systems, to a muscle work of feet, a back and stomach.

The results in push-up and lifting of a trunk in 30 seconds characterize the work in the high-speed and power mode of the hands' muscles, a shoulder girdle, and also abdominal muscle.

The complex characteristic of mobility in the shoulder joint gives "wringing" hands back. According to the results of an inclination it is possible to judge about the mobility in coxofemoral joints, and also about the flexibility of a spine column, elasticity and tensile properties of the sheaves and muscles participating in implementation of this test. Recognizing that manifestation of flexibility is mostly caused by tensile properties of the musculoskeletal device, there were chosen such exercises at which performance the movement would be made in the most important joints of the musculoskeletal device, and the largest muscular groups of a human body were exposed to extension. Besides, in the practice of physical education the greatest value has mobility in backbone joints, in coxofemoral joints and in the joints of the shoulder girdle, in which the amplitude of movements during the whole life tends to reduction. According to the researchers' point of view, a trunk inclination forward, costing on the straightened feet, can give the complex characteristic of a level of the general flexibility development (Vasiliev, 1965). There are a few facts proving its benefit:

First, performing an inclination forward the movements happen not in one, and in several and besides the largest and the most important joints of a human body, and the amplitude of movements in these joints during the whole life tends to decrease;

Secondly, this exercise characterizes tensile properties of the largest muscles of a human body - helps to unbend the back and hips;

Thirdly, the trunk inclination is a simple exercise on the coordination structure forward, it doesn't demand preliminary training and is available to the persons of different age, sex and physical fitness;

Fourthly, the inclination size can be easily determined with a sufficient accuracy by simple measuring adaptations in usual conditions of physical training process, and the results of measurements are expressed by the objective digital indicators and are evident for examinees;

Fifthly, the systematic use of an inclination gives forward the chance to have a regular control of the flexibility process, allows to estimate correctly expediency of the used means and methods.

The calculation of the reliability of the offered test tasks, which are presented in table 1 , showed a high degree of reliability of each test (within 0.900-0.978) that gives the chance to recommend them for an assessment of the separate parties of the female students' physical fitness.

According to the calculations, which are presented in the table, the correlation coefficient by the compound criterion has not so much differences from coefficient of multiple correlation. Both receptions of determination of the correlation's coefficient of the offered tests indicate that fact that three of them have good (0.80-0.85) and 
excellent (higher than 0.85) degree of informational content. There are: 10 minutes running $(\mathrm{r}=0.905, \mathrm{rt}=$ $0.850)$; lifting of a trunk $(\mathrm{r}=0.880, \mathrm{rt}=0.827)$; push-up in 30 seconds $(\mathrm{r}=0.815, \mathrm{rt}=0.805)$. Two tests have a low (lower than 0.60) degree of informational content. It is an inclination $(\mathrm{r}=0.377, \mathrm{rt}=0.515)$ and "wringing" $(\mathrm{r}=0.353, \mathrm{rt}=0.347)$. Nevertheless, the offered tests can be considered rather informative in respect of diagnostics. Practically there are used such diagnostics tests, which informational content is not less than 0,3 as there is no fixed test's informational content after which the test can be considered suitable. A lot of things are depended on a concrete situation. For the forecast there are used, as a rule, such tests, which informational content is higher-not less than 0.6 (Zatsiorsky, 1979, 1989).

The inter korrelyatsionny matrix, which is presented in table 2 , testifies to a high extent of communication between some signs. So the correlation coefficient between indicators in 10 minutes running and in lifting of a trunk in 30 seconds is equal 0.869 ; between the indicators in 10 minutes running and in push-up in 30 seconds - 0.834. It means that the development of the high-speed and power abilities of the hands' muscles, a shoulder girdle and an abdominal tension in a certain degree depends on the development of the general endurance. This fact can be explained to that results of test in lifting of a trunk and in push-up in 30 seconds are defined not only by the high-speed and power abilities, but also by the manifestation of endurance to high-speed and power efforts.

The correlation coefficient between the indicators in lifting of a trunk in 30 seconds and in push-up in 30 seconds, equaled 0.797, allows to make the conclusion that they adequately cause manifestation of the high-speed and power abilities of the not doing sports female students' organism. It is possible to make an assumption that the criteria defining the result at implementation of these tests are almost identical.

Though the correlation coefficient between indicators of weight and growth is equal 0.519 , nevertheless, in this case there is clearly traced the dependence of weight on growth of examinees against mass testing.

The correlation coefficient between the indicators of weight and 10 minutes running $(-0.340)$ belongs to the category of the low. Nevertheless, according to some information the experts in their researches explain the negative dependence of indicators on body weight with the assumption that in the exercises, demanding endurance in run, people with a small body weight has some advantage. This fact is explained by that with increase in a body weight the anaerobic opportunities of the examinees' organism, which are characterized by the maximum consumption of oxygen, raise more slowly, than a body weight. At the same time, the energy by performing the physical exercises increases in strict accordance with the body weight.

The correlation coefficients between indicators of weight and in lifting of a trunk in 30 seconds (-0.264), weight and in push-up in 30 seconds (-0.275) reflect (though it is insignificant) the negative dependence of high-speed and power abilities of the hands muscles, a shoulder girdle and muscles of an abdominal tension on the body weight.

It should be noted that the correlation coefficients between indicators of growth and indicators of physical fitness according to the offered tests are almost equal to zero that is explained by the lack of influence of the body length on some indicators of physical fitness, i. e. on manifestation of the studied motive qualities. It is remarkable that according to our calculations it isn't observed almost any interrelation between indicators of growth and the results by the performing an inclination. This fact confirms that the trunk inclination amplitude has a weak communication with body proportion indicators, especially forward at mass checks.

And the correlation coefficient between the indicators in an inclination and in "wringing" $(-0.331)$ also reflects the essential negative dependence of indicators of flexibility or mobility of joints of the body's various segments that confirms opinion of the experts considering flexibility as a complex quality in shoulders and that mobility of a spine column doesn't mean the same mobility in shoulder joints.

On the materials of the correlation analysis of the data research on the five offered tests and, including data of anthropometrical measurements (weight, growth), there was carried out the factorial analysis. As a result of calculation, according to the standard program (by the principle of the main components), there were allocated seven factors, which are presented in Table 3.

At interpretation of the allocated factors, first of all, there was considered the value of the received factorial weights. The names of the factors were selected so that they answered the nature of the parameters, having the greatest coefficients of correlation with the considered factor. Besides, their names were agreed with the other parameters having small values of coefficients.

The first factor has the biggest contribution to the selection dispersion $41.06 \%$. Significant factorial weights have tests here: 10 minutes running $(3.1)=-0.554$, lifting of a trunk in 30 seconds $(4.1)=-0.535$, push-up in 30 
seconds $(6.1)=-0.534$.

On the seventh factor significant factorial weights have also the "10 min. running" and "lifting of a trunk in 30 seconds" tests (respectively-0.795 and 0.552).

As these tests are connected with the endurance manifestation (running or general and high-speed and power belly muscles, muscles of hands and a shoulder girdle), so, these factors can be identified as an endurance. Endurance is one of these tests defining results by the performing. The general contribution of these factors to the generalized dispersion makes $-42.78 \%(41.06+1.72)$.

The second and the fifth factors are identified as anthropometry, as the greatest factorial weights fall here on indicators of weight $(0.602,0.725)$ and indicators of growth $(0.734-0.645)$. The general contribution of these factors to the generalized dispersion makes-26.28\% $(19.95+6.33)$. The factorial weights of the other indicators are little significant or close to zero.

The third and the fourth factors also have a clear boundary and are identified as flexibility and mobility in joints. Their contribution to the generalized dispersion of selection makes-29.22\% $(18.81+9.27)$.

The sixth of the allocated factors has a contribution to the generalized selection dispersion $-2.85 \%$ and are identified as the high-speed and power. The greatest factorial weights have the tests, which are intended for the assessment of the muscles' work of an abdominal tension, hands and a shoulder girdle in the high-speed and power mode here. There are also including lifting of a trunk in 30 seconds and push-up lying on the laps in 30 seconds. The specified tests have the corresponding factorial coefficients:-0.593 and 0.768 . The other coefficients are less significant and close to zero.

Thus, there are identified all factors allocated as a result of the factorial analysis of the offered set of the physical fitness' tests for a self-assessment of the non-doing sports female students' motive abilities (Palagina, 2005).

The total contribution of each area of manifestation of the students' motive abilities to the generalized dispersion makes:

1) The area of manifestation of the quality of endurance (the general and high-speed and power- $42.78 \%$ ).

2) The area of manifestation of the quality of flexibility and mobility in joints- $-29.22 \%$.

3) The area of manifestation of high-speed and power abilities of $2.85 \%$.

All other factors have low indicators of these factors' contribution to the generalized selection dispersion and by that they have no essential impact on indicators of examinees on the offered set of tests (Palagina, 2005).

It would be in addition unreasonable not to pay attention to the allocated factor "anthropometry" which total contribution to the generalized dispersion of selection made $26.28 \%$. Growth and weight have rather considerable communication with various motive manifestations and enter a component separately allocated factors.

So, on the basis of the presented results of the factorial analysis on the identification of the structure of the offered motive tests for an assessment of the female students' physical fitness at the age of 18-21 years, which aren't doing sports, it is possible to note that the considered complex of physical fitness' tests characterizes the following parties of physical fitness: high-speed and power, flexibility and mobility in joints, ability to perform the tasks for endurance.

\section{Conclusions}

The results of the conducted research on metrological justification of test "battery" for an assessment of the non-doing sports female students' physical fitness level, allowed to reveal:

1) The offered test "battery" for an assessment of the female students' physical fitness level rather fully characterizes the physical fitness level: 10 minutes running imposes certain requirements to the aerobic opportunities of an organism, to a condition of cardio respiratory and hormonal systems, to a muscle work of feet, a back and stomach; the results in push-up and lifting of a trunk for $30 \mathrm{sec}$. characterize work in the high-speed and power mode of the hands' muscles, a shoulder girdle, and also abdominal tension; the "wringing" hands gives the complex characteristic of mobility in shoulder joints and by the results of an inclination it is possible to judge mobility in coxofemoral joints, and also flexibility of a spine column, elasticity and tensile properties of the sheaves and muscles participating in implementation of this test.

Theoretical justification of this tests' complex showed that its performance covers various parties of physical fitness and by the results of its performance it is possible to judge a harmony of physical development and physical fitness. 
2) The factorial analysis of the presented complex of the physical fitness' tests, according to the factorial weights, characterizes the following parties of physical fitness: endurance, ability to perform the work in the high-speed and power mode, flexibility and mobility in joints.

3) The indicators of reliability of the used tests allow to apply them to an assessment of the separate physical abilities' development level, which are shown by the performing of the offered test tasks.

4) The high rates of informational test's content in 10 minutes running, lifting of a trunk and push-up in 30 seconds allow to recommend them for an assessment of the physical fitness level, and the tests for an assessment of the flexibility and mobility level—as supplementing this complex.

\section{References}

Akchurin, B. G. (1996). Problems of Optimization of the Higher School and Formation of Physical Health of Students: Thesis, Cand. of Science (p. 187). Ufa.

Bondarevskiy, E. Y. (1983). Informativeness of the tests used to characterize the physical fitness of the person. Theory and Practice of Physical Culture, 1, 23-25.

Gadzhiyev, S. (1986). Physical Education of the Female Students Taking into Account Regional Features of Physical Development and Readiness (on the example of regions AzSSR). Leningrad.

Godik, M. A., \& Zatsiorsky, V. M. (1965). Factorial analysis of experimental characteristics of any motive reactions. In Cybernetics and sport (p. 26). Moscow.

Hasin, L. A. (1995). Test to assess the physical fitness of students of institutes of physical culture. Theory and Practice of Physical Culture, 12, 43-48.

Hasin, L. A. (1999). The Test to Determine the Level of Physical Fitness of Student (7-17 years) (p. 74). Research Institute of Information Technologies of the Moscow State Academy of Physical Culture.

Kul'kov, V. V. (1980). Optimization of Physical Education of Students. Ivanovo.

Kustov, I. A. (1988). Increasing the Level of Physical Fitness of Girls 16-20 Years by Occupation Aquatic: Thesis, cand. ped. Science (p. 172). Moscow.

Kuzovenkov, V. V. (1982). Factors that determine the effectiveness of training students to perform standard set by the TRP in the women's 100m. Theory and Practice of Physical Culture, 6, 43-45.

Lapshina, G. G. (1989). Features of the female students' physical condition of the humanitarian faculty. Theory and Practice of Physical Culture, 4, 18-20.

Palagina, N. I. (2005). Optimization of the Students Physical Training on the Basis of an Assessment of Motive Abilities (p. 276). Joshkar-Ola.

Palagina, N. I., \& Polevshchikov, M. M. (2014). Metrological justification of motive tests for an assessment of the students' physical fitness level. In Physical Culture, Sport and Health (pp. 91-96).

Palagina, N. I. (2003). Study the effectiveness of the optimization of physical training of students on the basis of an assessment of motor abilities. In Physical Culture, Sport and Health (pp. 90-93). Yoshkar-Ola.

Palagina, N. I. (2006). The problem of optimization of physical training of students on the basis of assessment of motor abilities. Physical Culture, Sport and Health: Sat. Scientific (pp. 82-86). Art. Yoshkar-Ola.

Palagina, N. I., \& Blinova, M. L. (2010). The main directions of improvement of physical education of students. In Actual Problems of Improving and Adaptive Physical Education Students (pp. 181-186). Cheboksary.

Smirnov, Yu. I. (2000). Sports Metrology: Studies for the Student (p. 232). Moscow. Publishing center "Akademiya".

Vasiliev, E. P. (1965). Study of Flexibility of the Body and Experimental Substantiation of Means and Methods of Education. Riga.

Vinogradov, P. A. (1996). Social and Cultural Aspects of Health Promotion, Physical Culture and Olympism (p. 196). Moscow: Soviet Sport.

Yushkov, V. I. (1999). Ways of Improving the Physical Fitness of Students of Basic Training Department of the University: Thesis. Leningrad.

Zatsiorsky, V. M. (1989a). Fundamentals of Sports Metrology. Moscow.

Zatsiorsky, V. M. (1989b). Caution: Statistics. Theory and practice of physical culture, 2, 52-55. 


\section{Copyrights}

Copyright for this article is retained by the author(s), with first publication rights granted to the journal.

This is an open-access article distributed under the terms and conditions of the Creative Commons Attribution license (http://creativecommons.org/licenses/by/3.0/). 